\title{
ERRATUM
}

The Journal of Microbiology (2012) Vol. 50, No. 3, pp. 526-534

Copyright (c) 2012, The Microbiological Society of Korea

\section{Accumulation of Lipid Production in Chlorella minutissima by Triacylglycerol Biosynthesis-Related Genes Cloned from Saccharomyces cerevisiae and Yarrowia lipolytica}

\author{
Hsin-Ju Hsieh ${ }^{1 \star}$, Chia-Hung $\mathrm{Su}^{2}$, and Liang-Jung Chien ${ }^{2}$ \\ ${ }^{1}$ Material and Chemical Research Laboratories, Industrial Technology Research Institute; \\ 321 Kuang Fu Rd., Sec. 2, Hsinchu 30011, Taiwan \\ ${ }^{2}$ Graduate School of Biochemical Engineering, Ming Chi University of Technology; \\ 84 Gungjuan Rd., New Taipei 24301, Taiwan
}

In the article by Hsie et al. that appears in the Journal of Microbiology 2012; 50, 526-534. Page 526, The corresponding author for the article should be Liang-Jung Chien, Graduate School of Biochemical Engineering, Ming Chi University of Technology; 84 Gungjuan Rd., New Taipei 24301, Taiwan (ljchien@mail.mcut.edu.tw). 